\title{
Investigation of Obscured Flat Spectrum Radio AGN with the IceCube Neutrino Observatory
}

\author{
The IceCube Collaboration ${ }^{\dagger}$ \\ ${ }^{\dagger}$ http://icecube.wisc.edu/collaboration/authors/icrc17_icecube \\ E-mail: giuliano.maggi.olmedo@gmail.com
}

\begin{abstract}
The IceCube Collaboration recently reported the first detection of high-energy extraterrestrial neutrinos. Nonetheless, their origins are still unknown. In view of the IceCube discovery, active galactic nuclei (AGN) and gamma-ray bursts (GRBs) have been explored as possible sources, but no significant neutrino flux from these objects has been observed yet. The AGN that have previously been investigated by IceCube are characterized by a large gamma-ray flux as observed by dedicated instruments like the Fermi Space Telescope. In these bright AGN, neutrinos are expected to be generated via charged pion decay, as counterparts of the $\gamma$-rays produced by the decay of neutral pions. In contrast to the previously studied objects, it is also possible to target a specific class of AGN that show weak emission at the highest frequencies. These so-called "Obscured Flat Spectrum Radio AGN" show indications for a radio jet pointing towards Earth, as well as a column of matter in our line of sight that blocks high-energy electromagnetic emission from the central engine of the source. In addition to the blocked electromagnetic emission, a possible hadronic component will also be blocked by the obscuring material. This hadron beam dump is expected to give rise to an additional neutrino flux. Here we present results of a search for neutrino emission from selected AGN of this type using IceCube data.
\end{abstract}

Corresponding authors: Giuliano Maggi, Krijn D. de Vries, Nick van Eijndhoven*

Vrije Universiteit Brussel, Dienst ELEM, IIHE, Pleinlaan 2, 1050 Brussels, Belgium

35th International Cosmic Ray Conference - ICRC2017

10-20 July, 2017

Bexco, Busan, Korea

* Speaker. 


\section{Introduction}

Several neutrino astronomy analyses have been performed in order to find the origin of astrophysical high-energy ( $\sim \mathrm{TeV}-\mathrm{PeV})$ neutrinos as the ones discovered by IceCube [1,2]. Nevertheless, so far no significant evidence has been reported for a link between high-energy neutrino emission and a certain astrophysical source. The main source candidates for high-energy neutrino emission belong to two classes of astrophysical objects, Gamma Ray Bursts (GRBs) and Active Galactic Nuclei (AGN).

The main characteristic of the investigated subclasses of AGN [3, 4, 5] is that they have a large $\gamma$-ray flux as observed by dedicated telescopes. In this scenario, the neutrino production is expected to be dominated by the interaction of accelerated protons with ambient photons $(p \gamma)$ in the AGN environment. These interactions produce neutrinos via the decay of various charged mesons, where the decay of neutrons and muons can also contribute to the neutrino production (see for example [6]). The $\gamma$-ray flux could have a hadronic origination, which is dominated by the decay of various neutral mesons that are produced as a consequence of the $p \gamma$ interaction. Therefore, one can argue that the larger the charged meson multiplicity is, the larger is the neutral meson production, and consequently the neutrino - $\gamma$-ray correlation can be established.

The efficiency of the expected neutrino production in bright AGN via $p \gamma$ interactions depends on several factors, as for example, the photon field density in the AGN environment, and the fraction of $p \gamma$ interactions that indeed produces neutrinos [7]. However, another channel to produce high-energy neutrinos in AGN could be via hadronuclear interactions $(p p)[6,8]$. In this context, the existence of hidden Cosmic-Ray accelerators has been proposed [9], where by comparing the neutrino flux detected by IceCube with the $\gamma$-rays observed with the Fermi telescope, a tension is observed, if one considers only neutrino production from $p \gamma$ interactions.

In this work, we present the results of a search for high-energy neutrinos $\left(v_{\mu}+\bar{v}_{\mu}\right)$ with the IceCube neutrino observatory from a specific class of AGN, called "Obscured Flat Spectrum Radio AGN" (OFSR-AGN) [10]. OFSR-AGN, that fall into the category of hidden cosmic-ray accelerators, show indications of a possible radio jet which is pointing towards Earth and a surrounding column of matter in our line of sight, which blocks the electromagnetic emission at the highest frequencies, as in the $X$-ray domain. Therefore, instead of being bright objects, OFSR-AGN are opaque at short wavelengths, whereas emission of long wavelengths, as radio, is not affected by the obscuring material. If hadronic acceleration takes place at OFSR-AGN, neutrino production is expected via the "standard" $p \gamma$ interaction. However, a second component is expected to enhance the neutrino production in OFSR-AGN via hadron-column of matter interactions. The hadrons that interact with matter are expected to be the primary ones that did not interact with the ambient photons, or the ones that follow from the $p \gamma$ interactions.

The current analysis is based on the investigation of 14 OFSR-AGN with four years of data collected at the IceCube neutrino observatory at the South Pole, between 2012 April 26 and 2016 May 20. We specifically search for muons induced by muon-neutrino $\left(v_{\mu}+\bar{v}_{\mu}\right)$ interactions in or nearby the IceCube detector, because these provide the best directional information. Our data as obtained at the South Pole is dominated by atmospheric muons, nonetheless, after several event selection procedures, we obtain an irreducible atmospheric neutrino event rate. Subsequently, to distinguish atmospheric neutrinos from astrophysical neutrinos, we use an unbinned likelihood 
method as described in [11]. The results obtained from the current analysis show no significant neutrino emission from the investigated population of OFSR-AGN. We present a $90 \%$ upper limit for each investigated source, for an $E^{-2}$ power-law neutrino emission spectrum.

\section{Obscured Flat Spectrum Radio AGN}

OFSR-AGN are characterized by having a flat spectrum radio emission, and by being obscured by a column of matter that blocks our line of sight at frequencies in the $X$-ray domain. The latter could be a consequence of a tilted AGN torus, a misaligned disc or any column of matter in the AGN environment that causes the AGN obscuration phenomenon [12, 13].

An OFSR-AGN catalog based on radio and $X$-ray observations was constructed in [10]. The selection procedure started from an initial sample of 735 objects obtained from the second catalog of AGN detected by Fermi-LAT (2LAC) [14], and from a catalog of strong radio-emitting galaxies (referred to as the Nijmegen radio catalog) [15]. A first selection was made by selecting objects that are not expected to have an $X$-ray flux attenuation due to intergalactic medium or redshift effects. After applying a redshift selection $z<0.17$, the initial sample of 735 objects is reduced to 209 sources.

In OFSR-AGN, a particle emission is required to be directed towards Earth, consequently, we require that the AGN radio jet points towards the observer, which is usually indicated by a flat radio spectrum. As outlined in [10], this can be determined by the value of the radio spectral index $\alpha_{R}$ in the power law $F_{v} \propto v^{\alpha_{R}}$, where objects with $\alpha_{R}>-0.5$ are typically classified as Flat Spectrum Radio Quasars or BL Lac objects. After selecting objects with $\alpha_{R}>-0.5$, a total of 98 sources remain.

In order to discriminate OFSR-AGN from non obscured AGN, the luminosity intensity at a frequency of $v_{X-\text { ray }}=3.03 \cdot 10^{17} \mathrm{~Hz}$ is analyzed, as explained below. Using the NASA/IPAC Extragalactic Dataset (NED) [16], 62 objects out of 98 sources have measurements at $v_{X \text {-ray. }}$.

As outlined in [10], for the remaining 62 objects, three source categories are distinguished according to various signatures in the electromagnetic domain. The categories are Flat Spectrum Radio Quasars (FSRQs) (14), BL Lacertae (BL Lac) objects (45) and Ultra Luminous Infrared Galaxies (ULIRGs) (3).

In the FSRQ sample composed of 14 sources, the galaxy 3C273 is excluded from the analysis because of the exceptionally strong $X$-ray and radio emission, which implies that $3 \mathrm{C} 273$ does not qualify as an obscured source.

The ULIRG sample is composed of 3 objects, including the galaxy NGC 3628, also known as the "Hamburger Galaxy". For this galaxy, the $X$-ray measurements given in NED are unsuitable for further analysis of the corresponding $X$-ray frequency. The large uncertainties of the two fluxes given by Chandra and XMM telescopes as presented in [16], make these measurements compatible with background, which would imply that these space telescopes did not have the capability to detect a clear signal at $v_{X \text {-ray }}=3.03 \cdot 10^{17} \mathrm{~Hz}$, or indeed this object has a very low $X$-ray emission. However, the latter fact in addition to detailed telescopic views as shown in Fig. 1, make it worthwhile to investigate this object as a possible neutrino source powered by proton-matter interactions. Consequently, this object was also studied using IceCube data. 


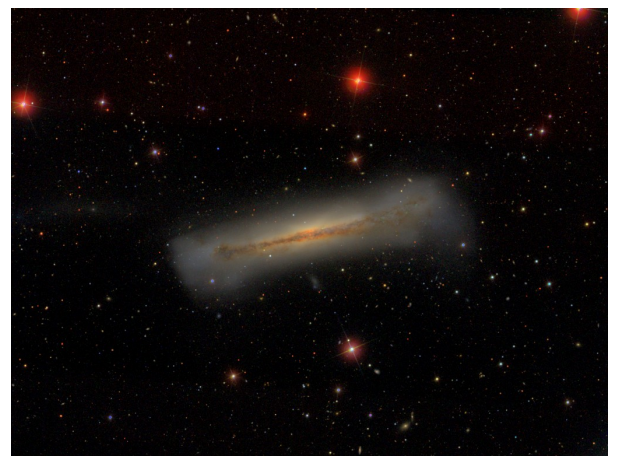

Figure 1: The galaxy NGC 3628, which is categorized as an Ultra Luminosity Infra Red Galaxy (ULIRG) in [10]. Credits: [16]

After excluding the galaxy 3C273, and the separate treatment of NGC 3628, 60 objects remain, which are grouped in two categories, BL Lac and FSRQ-ULIRG. The latter contains FSRQ and ULIRG galaxies, which are treated in a similar way due to the similarity in radio and $X$-ray emission. To determine whether a source has low emission compared with objects in the same category, source intensities at $v_{X \text {-ray }}$ are compared.

To avoid the classification of OFSR-AGN as a consequence of low $X$-ray emission due to a weak central engine instead of a weak $X$-ray emission due to absorption by a column of matter in our line of sight, the $X$-ray luminosity is normalized to the radio luminosity for the FSRQ-ULIRG category. For objects in the BL Lac category, no correlation between $X$-ray and radio is observed [10], therefore, no further normalization is applied to the $X$-ray intensity of objects in the BL Lac category.

Considering the two categories and a normalization factor for FSRQ-ULIRG objects, the intensity $I$ for the selection of OFSR-AGN is given by $I_{B}=L_{X \text {-ray }}$ for objects in BL Lac category, and $I_{F-U}=L_{X-\text { ray }}^{\beta} / L_{\text {radio }}$ for objects classified as FSRQ-ULIRG, where $\beta$ is the normalization factor as computed in [10], $L_{X \text {-ray }}$ is the $X$-ray luminosity at $3.03 \cdot 10^{17} \mathrm{~Hz}$ and $L_{\text {radio }}$ is the radio luminosity at $1.4 \cdot 10^{9} \mathrm{~Hz}$. The final procedure to select OFSR-AGN considers the objects that have the lowest intensity $I$ in each population, which are taken from the lowest quartile in each population [10].

The list of resulting OFSR-AGN objects as selected in [10], is presented in Table 1 together with the results obtained in the current work, which will be described later on.

\section{The IceCube Detector and the Data Sample}

The IceCube neutrino observatory is a cubic kilometer detector located at the geographic South Pole [18], which is buried 1450 meters under the ice surface (called InIce detector). The instrumented volume consists of an array of 86 vertical strings, where the data collection is performed by 5160 Digital Optical Modules (DOMs) that are attached to the strings. These DOMs are placed between 1450 and 2415 meters under the ice surface.

The horizontal string spacing corresponds to $\sim 125$ meters, except for a denser core where the spacing amounts to about 70 meters. This denser IceCube instrumentation is called DeepCore, 
which differs from the rest of the detector in order to perform neutrino studies at lower energy. The vertical spacing between DOMs in the DeepCore array corresponds to $\sim 7$ meters, whereas the spacing in the rest of the instrumentation is $\sim 17$ meters. Furthermore, the IceCube observatory has a dedicated instrumentation for studying cosmic-ray interactions with the terrestrial atmosphere. The latter is an array composed of 162 tanks of ice, located on the ice surface, which is called IceTop detector.

The IceCube event rate at South Pole amounts to $\sim 2700 \mathrm{~Hz}$, which is highly dominated by atmospheric muons induced by cosmic-ray interactions with the terrestrial atmosphere. An IceCube event (InIce) is defined when at least four pairs of neighboring DOMs are fired within a time window of $5 \mu \mathrm{s}$. If this condition to create an event is fulfilled, the times of the first $\left(t_{\text {init }}\right)$ and last $\left(t_{\text {last }}\right)$ trigger pulse are extended to read out the whole event in a time window of: $\left[t_{\text {init }}{ }^{-}\right.$ $\left.10 \mu \mathrm{s}, t_{\text {last }}+22 \mu \mathrm{s}\right][19]$.

After a first data processing at the South Pole to reject atmospheric muon events in the IceCube detector, the event rate is reduced to $\sim 600 \mathrm{~Hz}$. These events are then transmitted to the North via satellite, where further reconstructions and event selections are performed as in [4], until a final sample dominated by neutrinos with a rate on the order of $2.5 \mathrm{mHz}$ is obtained. These events are predominantly atmospheric neutrino events, with a small contribution $\lesssim 1 \%$ estimated to be astrophysical neutrinos.

In the current analysis, we have used an energy estimator to improve the angular resolution, which acts as a "weight" for determining how likely the recorded pulses are created by a certain track hypothesis. This slight improvement in the angular resolution with respect to the one used in [4], is enhanced by the likelihood formula used in the analysis method as described in Section 4, which results in an improved sensitivity.

The sample used for this analysis contains tracks with a declination $\delta>-5^{\circ}$, such that we cover the region of the sky where the 14 OFSR-AGN are located. The total number of events in this IceCube data sample amounts to 315639 events, collected in 1414.7 days (2012-2015), that implies an event rate of $2.58 \mathrm{mHz}$.

\section{Analysis Method}

In order to discriminate events caused by atmospheric neutrinos from their astrophysical counterpart, we apply the statistical method described in [11]. This is based on an unbinned likelihood ratio, where the likelihood function is given by,

$$
\mathscr{L}\left(\vec{x}_{s}, n_{s}, \gamma\right)=\prod_{i}^{N}\left(\frac{n_{s}}{N} \mathscr{S}_{i}+\left(1-\frac{n_{s}}{N}\right) \mathscr{B}_{i}\right),
$$

where $x_{s}$ is the source position, and $n_{s}$ and $\gamma$ are the best-fit values for the number of signal events and spectral index due to a neutrino source located at $x_{s}$. We will refer to $\gamma$ as $\gamma_{v}$. The expressions $\mathscr{S}$ and $\mathscr{B}$ correspond to the signal and background probability density functions respectively, and $N$ indicates the total number of events (signal plus background). The details of Eq. 4.1 can be found in [11].

The test statistic can be constructed from the likelihood function in Eq. 4.1, which can be written as, 


$$
\lambda=2 \cdot \operatorname{sign}\left(n_{s}\right) \log \left[\frac{\mathscr{L}\left(\vec{x}_{s}, n_{s}, \gamma_{v}\right)}{\mathscr{L}\left(\vec{x}_{s}, 0\right)}\right],
$$

where the larger $\lambda$ is, the less compatible the observation is with the background only hypothesis.

The fit for the spectral index of the signal can help to distinguish astrophysical signal from atmospheric background, if the source energy spectrum is considerably harder than the background (approximately $E^{-3.7}$ ). In the present analysis, we do not assume any particular model about the spectral index of the signal, so we let $\gamma_{v}$ vary as a free parameter in the range $1 \leq \gamma_{v} \leq 4$.

\section{Results}

We perform the likelihood search method at each of the source locations in the catalog of OFSR-AGN objects, calculating $\lambda$ for the choice of $n_{s}$ and $\gamma_{v}$ that maximize the value of the likelihood function. We estimate the significance (p-value) of each result by performing the same analysis on the data when it is scrambled in right ascension, and counting the fraction of such scrambled trials which yield a $\lambda$ more significant than the one obtained for the real data. The results obtained from the IceCube search for high-energy neutrino emission $\left(v_{\mu}+\bar{v}_{\mu}\right)$ from OFSRAGN, show that there is no significant evidence that indicates high-energy neutrino production at the investigated sources.

The search for $v_{\mu}+\bar{v}_{\mu}$ inducing a muon as a consequence of a neutrino charged current interaction in or nearby the IceCube detector, was performed using four years of IceCube data. We have set a $90 \%$ upper limit $\Phi^{90 \%}$ on the neutrino flux $\left(v_{\mu}+\bar{v}_{\mu}\right)$ emitted from each source in the OFSRAGN sample, as displayed in Table 1. This was calculated assuming a power-law of $\frac{d \Phi_{v}}{d E} \propto E^{-2}$ for energies between $1 \mathrm{TeV}-1 \mathrm{PeV}$. Furthermore, in Table 1, we present the pre-trial p-values, the best-fitting spectral index $\gamma_{v}$ and the number of signal-like events $n_{s}$ as provided by the maximization of the likelihood formula. In addition, we give the position (declination $\delta$ and right ascension ra) of each source.

As shown in Table 1, the two most significant pre-trial p-values are 0.034 and 0.037 , which correspond to the objects NGC 3628 and 2MASXJ05581173+5328180 respectively. For the more significant source, NGC 3628, we determine the post-trial p-value, which amounts to 0.3 . Nevertheless, since we obtain two sources with approximately equal pre-trial p-values, we also determine an a-posteriori p-value. This a-posteriori p-value reflects the probability of finding 2 out of 14 sources with such a p-value to be 0.08 . This is also compatible with background.

\section{Discussion}

The IceCube investigation of 14 sources classified as Obscured Flat Spectrum Radio AGN shows no significant evidence for neutrino emission from the analyzed astrophysical objects. The two most significant sources belong to the same category (FSRQ-ULIRG), these galaxies are NGC 3628 and 2MASXJ05581173+5328180, where the former is classified as an ULIRG and the latter as an FSRQ.

Moreover, the two sources with the largest significance have been investigated in the Nijmegen radio catalog as possible ultra-high-energy cosmic-ray emitters [15]. In this catalog, four groups of 


\begin{tabular}{|l|l|l|l|l|l|l|l|}
\hline Source name & $\mathrm{ra}\left(^{\circ}\right)$ & $\delta\left(^{\circ}\right)$ & $\mathrm{p}$-val & $n_{s}$ & $\gamma_{v}$ & $\Phi^{90 \%}$ & Object-Class \\
\hline 2MASXJ05581173+5328180 & 89.55 & 53.47 & 0.037 & 16.12 & 2.73 & 1.08 & FSRQ-ULIRG \\
MRK0668 & 211.75 & 28.45 & 0.300 & 2.48 & 3.76 & 0.88 & FSRQ-ULIRG \\
NGC3628 & 170.07 & 13.59 & 0.034 & 6.88 & 2.21 & 0.72 & FSRQ-ULIRG \\
ARP220 & 233.74 & 23.50 & 1.0 & 0.0 & 4.0 & 0.75 & FSRQ-ULIRG \\
3C371 & 271.71 & 69.82 & 0.242 & 5.35 & 4.0 & 1.18 & BL Lac \\
4C+04.77 & 331.07 & 4.67 & 0.412 & 0.73 & 2.05 & 0.65 & BL Lac \\
1H1720+117 & 261.27 & 11.87 & 1.0 & 0.0 & 3.11 & 0.70 & BL Lac \\
B21811+31 & 273.40 & 31.74 & 0.076 & 11.93 & 2.85 & 0.85 & BL Lac \\
CGCG186-048 & 176.84 & 35.02 & 1.0 & 0.0 & 2.88 & 0.86 & BL Lac \\
GB6J1542+6129 & 235.74 & 61.50 & 1.0 & 0.0 & 2.68 & 1.07 & BL Lac \\
PKS1717+177 & 259.80 & 17.75 & 0.142 & 7.81 & 2.97 & 0.75 & BL Lac \\
RGBJ1534+372 & 233.70 & 37.27 & 0.318 & 3.95 & 3.05 & 0.90 & BL Lac \\
SBS0812-578 & 124.09 & 57.65 & 0.386 & 1.70 & 3.84 & 1.09 & BL Lac \\
SBS1200+608 & 180.76 & 60.52 & 1.0 & 0.0 & 2.46 & 1.09 & BL Lac \\
\hline
\end{tabular}

Table 1: List of sources in the AGN population and the their position $(\mathrm{ra}, \delta)$ in Equatorial coordinates in degrees. The following values correspond to the fit result that are obtained by maximizing the likelihood formula. Here we show the pre-trial p-value, the best fit number of signal events $n_{s}$ and the spectral index $\gamma_{v}$. The upper limit on the $v_{\mu}+\bar{v}_{\mu}$ flux $\Phi^{90 \%}$ is given in units of $10^{-12}\left[\mathrm{TeV} \mathrm{cm}{ }^{-2} \mathrm{~s}^{-1}\right]$.

galaxies have been investigated which have been constructed according the source morphology and observations in the electromagnetic domain. These groups are: Starforming galaxies, Jets \& Lobes, Point sources and Unknown morphology. The galaxy 2MASXJ05581173+5328180 is classified as a Point source, whereas NGC 3628 belongs to the Starforming galaxy category.

In view of the current results, a follow up study could be made by investigating ULIRG objects, Starforming galaxies and Point Source, where the last two would belong to a classification as made in the Nijmegen radio catalog [15]. This study can be accomplished by performing stacking analyses of the same class of objects. Moreover, in order to obtain more sources, an investigation on how to correct the observed flux for far away objects would be needed, since the redshift selection was the hardest cut that was used to construct the OFSR-AGN catalog [10]. On the other hand, to have a conception about the expected neutrino spectral index from OFSR-AGN could help on the search for neutrinos from these kind of sources, which implies a full modeling of the $p \gamma$ and $p p$ interactions, including secondary hadrons propagation through the AGN medium.

\section{References}

[1] IceCube Collaboration, M. G. Aartsen et. al, Science 342, 1242856 (2013).

[2] IceCube Collaboration, M. G. Aartsen et. al, Phys. Rev. Lett. 113, 101101 (2014).

[3] IceCube Collaboration, M. G. Aartsen et. al, Astrophys. J. 796, 109 (2014). 
[4] IceCube Collaboration, M. G. Aartsen et. al, Astrophys. J. 835, 151 (2017).

[5] IceCube Collaboration, M. G. Aartsen et. al, Astrophys. J. 807, 46 (2015).

[6] Gaisser T. and Stanev T., Astroparticle Physics 39-40, 120-128 (2012).

[7] Reimer A., Journal of Physics: Conference Series 355, 012011 (2012).

[8] Murase K. et. al, Phys.Rev.D 88, 121301 (2013).

[9] Murase K. et. al, Phys.Rev.Lett. 116, 071101 (2016).

[10] Maggi G. et. al, Phys.Rev.D 94, 103007 (2016).

[11] Braun J. et. al, Astropart. Phys. 29, 299-305 (2008).

[12] Lawrence A. and Elvis M., The Astrophysical Journal 714, 1 (2010).

[13] Bianchi S., Maiolino R. and Risaliti G., Advances in Astronomy 2012, Article ID 782030, 17 pages, (2012).

[14] Fermi-LAT Collaboration, M. Ackermann et. al, The Astrophysical Journal 743, 2 (2011).

[15] van Velzen S. et. al, A\&A 544, 19 (2012).

[16] NASA/IPAC Extragalactic Database (NED), https://ned.ipac.caltech.edu

[17] Netzer H., "The Physics and Evolution of Active Galactic Nuclei”, Cambridge University Press (2013).

[18] IceCube Collaboration, M. G. Aartsen et. al, JINST 12, P03012 (2017).

[19] IceCube Collaboration, M. G. Aartsen et. al, Astropart. Phys. 78, 1-27 (2016). 